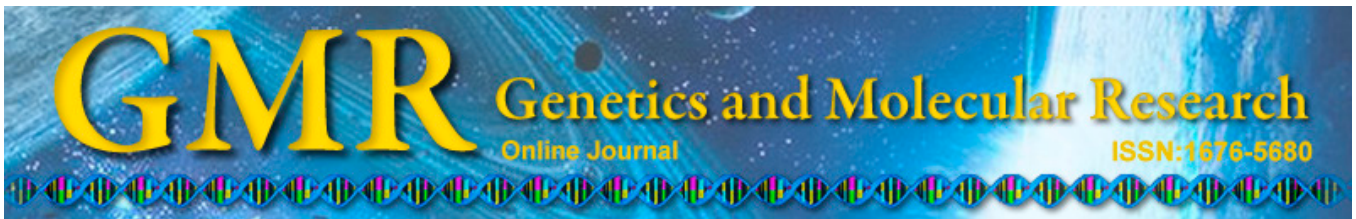

\title{
Application of indirect linkage analysis and direct genotyping to hemophilia A carrier detection in Sichuan, China
}

\author{
P. Sun, L. Ma, G. Diao, C.Q. Li and F.Z. Lin \\ Blood Biochemistry and Molecular Biology Research Center, \\ Institute of Blood Transfusion, Chinese Academy of Medical Sciences, \\ Chengdu, Sichuan, China \\ Corresponding author: F.Z. Lin \\ E-mail: lfangzhao@126.com / sunpan_1@163.com
}

Genet. Mol. Res. 14 (3): 8229-8235 (2015)

Received September 5, 2014

Accepted March 18, 2015

Published July 27, 2015

DOI http://dx.doi.org/10.4238/2015.July.27.10

\begin{abstract}
Hemophilia A (HA) is an inherited X-linked bleeding disorder caused by mutations in the factor VIII gene. Prenatal detection in female carriers from families with HA is important to reduce the number of HA patients. The purpose of this study was to detect carriers in families with HA from Sichuan, China, using linkage analysis and a direct genotyping method. A total of 18 HA families were studied. Using a combination of intron 22 inversion, intron 1 inversion, the $B c l$ I polymorphic site in intron 18 , the HindIII polymorphic site in intron 19, and dinucleotide CA-repeat markers in introns 1, 13, 22, and 24, we were able to detect HA in $88.9 \%$ (16/18) of the families studied. HA was detected in the remaining two families by direct genotyping. This study gave the participants a good understanding of their genetic condition and gave us a preliminary understanding of the prevalence of each mutation in Sichuan HA patients.
\end{abstract}

Key words: Hemophilia A; Carrier detection; Linkage analysis; Direct genotyping 


\section{INTRODUCTION}

Hemophilia A (HA) is an inherited X-linked bleeding disorder caused by mutations in the factor VIII (FVIII) gene. It affects approximately 1/5000 males, while females are at risk of being carriers (Oldenburg et al., 2004). Hemophilia A is usually classified according to the plasma concentration of FVIII clotting activity (FVIII:C), as severe ( $<1 \%$ of normal), moderate $(1-5 \%)$, or mild $(5-25 \%)$. It is important to detect the female carriers in families with HA to reduce the number of HA patients through prenatal screening. The FVIII gene is $186 \mathrm{~kb}$ in length and has 26 exons ranging in size from $50 \mathrm{bp}$ to $3.2 \mathrm{~kb}$ (Gitschier et al., 1984). The most common mutation of the FVIII gene is the inversion of intron 22, accounting for about half of severe HA cases (Lakich et al., 1993; Naylor et al., 1993). The second most common mutation may be the inversion of intron 1, with a prevalence of $4.8 \%$ in British severe HA patients (Tizzano, 2003). Nearly every HA family has an individual FVIII mutation without the inversion (The hemophilia A mutation, structure, test and resource site, www.factorviii-db.org). Therefore, it is time-consuming and expensive to directly determine the mutation for these families. Linkage analysis has been widely used for genetic diagnosis of HA. The indirect method is fast and inexpensive to perform. The most useful linkage markers are restriction fragment length polymorphism (RFLP) and short tandem repeat (STR) markers situated close to the gene or within its introns (Lalloz et al., 1991; Peake et al., 1993).

In this study, we applied both linkage analysis and direct genotyping to carrier detection in 18 HA families from Sichuan, China. The process gave them a good understanding of their genetic condition and gave us a preliminary understanding of the prevalence of each mutation in Sichuan HA patients.

\section{MATERIAL AND METHODS}

\section{Patients, samples, and DNA extraction}

A total of 18 HA families, including 32 female relatives, from Sichuan Province, China, were investigated in this study. Patients and their relatives were fully informed and signed agreements prior to participating in the genetic studies. In some cases, a signed agreement was obtained from the parents or legal representatives of the patient. The FVIII:C was measured in a standard one-stage clotting assay using a CA-1500 Hemostasis Analyzer (Sysmex, Kobe, Japan) with coagulation factor-deficient plasma (Sysmex).

Total genomic DNA was extracted from peripheral blood leukocytes using a blood DNA extract kit (Omega, USA). The purity, quality, and concentration of DNA were assessed by ultraviolet spectroscopy at 260 and $280 \mathrm{~nm}$. DNA samples were stored at $-20^{\circ} \mathrm{C}$ until required for testing.

\section{Detection of intron 22 and intron 1 inversions}

All samples were screened for the inversion analysis. Both intron 1 and intron 22 inversions were detected by polymerase chain reaction (PCR), as reported previously (Liu et al., 1998; Bagnall et al., 2002). The following modification was made to the intron 22 PCR: only primers $\mathrm{P}, \mathrm{B}$, and $\mathrm{Q}$ were used to simplify the amplification, and two amplification reactions were made for every sample (one with primers $\mathrm{P}$ and $\mathrm{B}$, the other with $\mathrm{P}$ and $\mathrm{Q}$ ). People with 
intron 22 inversion were positive for PB, normal people were positive for PQ, and female carriers were positive for both.

\section{Linkage analysis}

\section{RFLP analysis}

The $B c$ II polymorphic site in intron 18 and HindIII in intron 19 were studied by RFLP, as described previously (Tasleem Raza et al., 2009). Briefly, for BclI and HindIII, samples were amplified in a $20-\mu \mathrm{L}$ reaction mixture containing $2.5 \mathrm{mM} \mathrm{MgCl}_{2}, 0.2 \mathrm{mM}$ dNTPs, 1.0 $\mathrm{mM}$ of each primer, and 1.0 U Taq DNA polymerase. Samples were denatured at $95^{\circ} \mathrm{C}$ for 5 min prior to temperature cycling $\left(30\right.$ cycles, denaturation at $95^{\circ} \mathrm{C}$, annealing at $58^{\circ} \mathrm{C}$ and extension at $72^{\circ} \mathrm{C}$, for $30 \mathrm{~s} \mathrm{each}$, and a final extension at $72^{\circ} \mathrm{C}$ for $5 \mathrm{~min}$ ). The PCR products were digested by the appropriate enzymes and then electrophoresed on $2 \%$ agarose gel in $1 \% \mathrm{X}$ TAE buffer (Tris base, acetic acid, and ethylenediaminetetraacetic acid) at $100 \mathrm{~V}$ for $40 \mathrm{~min}$. Bands were visualized using EvaGreen dye (Bio-Rad, Hercules, CA, USA).

\section{STR analysis}

Dinucleotide CA-repeat markers in introns 1, 13, 22, and 24 were studied in all samples. Primer name, sequence, fluorescent dye, and amplified product size are given in Table 1. Samples were amplified in $20 \mu \mathrm{L}$ of the same reaction mixture described above. PCR amplification was carried out by small modifications. The PCR products were then analyzed on an automated fluorescent DNA sequencer (ABI 3730, USA). Analysis was carried out using GeneScan 3.7 and genotyper $3.7(\mathrm{ABI})$.

Table 1. Summary of primer name, sequence, fluorescent dye, and polymerase chain reaction product size.

\begin{tabular}{llc}
\hline Primer & Sequence $\left(5^{\prime}-3^{\prime}\right)$ & Allele size $(\mathrm{bp})$ \\
\hline Intron 1-F & (FAM)TGATCCAGCAACTCGACTTCT & 195 \\
Intron 1-R & TCTTGGTCTGCCTTCTGACT & 190 \\
Intron 13-F & (FAM)CGCTCCATTGTTTCTACTTGCA & 178 \\
Intron 13-R & GCCTAGAGAATGCCAAAGTAACAC \\
Intron 22-F & (HEX)CAAGGTGTCAAATCCCACGT & 136 \\
Intron 22-R & TATCCTCCACCCTGGTCTCA & \\
Intron 24-F & (FAM)AGTCCAAGATCAAGGGGTAGG & \\
Intron 24-R & CATCACATTCCAGCCTGGACT & \\
\hline
\end{tabular}

\section{Direct sequencing}

The families that were not diagnosed by intron inversions, RFLP, or STR analysis were direct sequenced for the coding region. We also randomly selected five families to confirm the diagnoses by the indirect method. All the exons of the FVIII gene were amplified with the primers described by He et al. (2013). 


\section{RESULTS}

\section{Clinical classification}

According to FVIII:C levels, 17 of the 18 patients were classified as moderate (1-5\%), and the remaining patient was mild (11.7\%).

\section{Inversion detection}

We first screened FVIII intron 22 inversions; eight patients of 18 cases (44\%) were positive for that mutation. We then made carrier diagnoses for the families with intron 22 inversions; 15 female relatives in those families were carriers with the mutation, and nine were negative for it. Intron 1 inversion screening revealed that none of the patients in our study was positive for that inversion.

\section{Linkage analysis}

We adopted six linkage markers to make carrier diagnosis for HA: the $B c l$ I polymorphic site in intron 18; the HindIII site in intron 19; and the dinucleotide CA-repeat markers in introns $1,13,22$, and 24. All the families were analyzed using this method, and only the families with a heterozygous mother could be diagnosed. The results are shown in Table 2.

Table 2. Number of families that could be diagnosed and female carrier status of each linkage marker.

\begin{tabular}{lcc}
\hline Linkage marker & $\begin{array}{c}\text { Number of families that could be diagnosed } \\
\text { (\% of the total) }\end{array}$ & Female carrier status \\
\hline BcII & $5(26.3 \%)$ & 10 carriers, 6 normal \\
HindIII & $3(15.8 \%)$ & 5 carriers, 6 normal \\
STR1 & $5(27.7 \%)$ & 8 carriers, 13 normal \\
STR13 & $7(36.8 \%)$ & 11 carriers, 7 normal \\
STR22 & $2(10.5 \%)$ & 5 carriers, 5 normal \\
STR24 & 0 & 0 \\
\hline
\end{tabular}

\section{Direct sequencing}

Direct sequencing of the coding region of the FVIII gene was conducted in seven HA families. Mutations were identified in six families; three located in exon 14, the other three located in exons 3, 13, and 24. No mutation was identified in one HA patient. The sequencing results of all mutations are shown in Figure 1 and Table 3. The nucleotide number of the FVIII mutation was assigned according to the cDNA sequence (GenBank accession No. NM_000132.2) starting with A of the initiator ATG site as +1 . The amino acid sequence numbering assigns the first residue of mature FVIII as +1 according to the Haemophilia A Mutation, Search, Test and Resource Site (HAMSTeRS) nomenclature that has been widely used in other FVIII studies. 

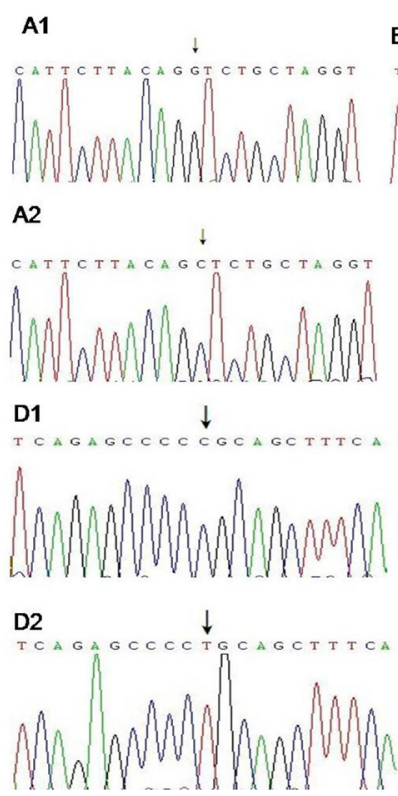

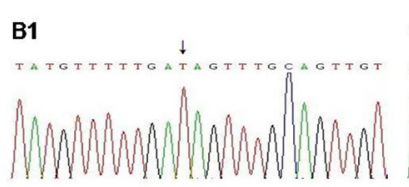

B2

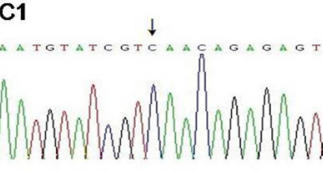

C2

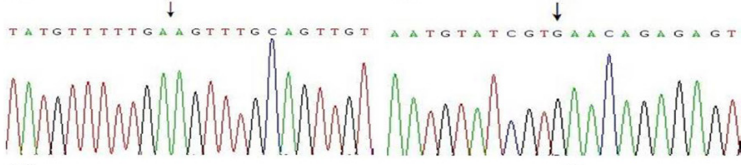

E1
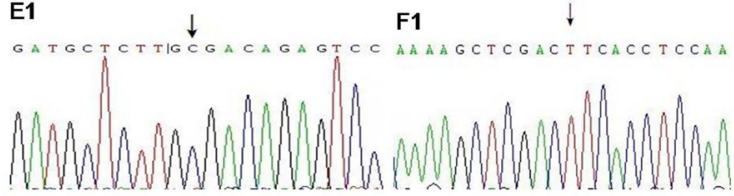

E2 $\downarrow$

lGaCAGAGTCO

F2

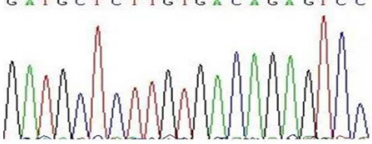

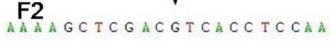

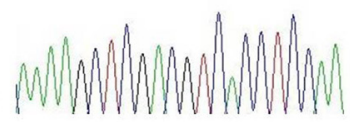

Figure 1. Sequencing results for all mutations. A1-F1: sequence of normal FVIII gene. A2-F2: sequence of affected FVIII gene.

\section{Table 3. Mutations of FVIII gene identified in this study.}

\begin{tabular}{lllll}
\hline Exon & Mutation & Type of mutation & Amino acid substitution & Female carrier status \\
\hline 3 & c. $70 \mathrm{G}>\mathrm{C}$ & Missense mutation & Gly70Ala & 2 carriers, 1 normal \\
13 & c. $642 \mathrm{delT}$ & Frameshift mutation & Asp642/Stop659 & 2 carriers, 4 normal \\
14 & c. $985 \mathrm{C}>\mathrm{G}$ & Nonsense mutation & Ser985Stop & 2 carriers, 1 normal \\
14 & c. $1708 \mathrm{C}>\mathrm{T}$ & Missense mutation & Arg1708Cys & 2 normal \\
14 & c. $814 \mathrm{C}>\mathrm{T}$ & Nonsense mutation & Arg814Stop & 1 carrier, 1 normal \\
24 & c. $2229 \mathrm{C}>\mathrm{T}$ & Missense mutation & Leu2229Arg & 3 carriers \\
\hline
\end{tabular}

\section{DISCUSSION}

The actual prevalence of HA in China is still not well known. The molecular analysis of the HA gene was carried out to develop mutational analysis that could help in carrier detection and prenatal diagnosis. We aimed to establish a good strategy for HA carrier detection in Sichuan Province. It has been reported that intron 22 inversion is the most common mutation, with a prevalence of $45-50 \%$ in severe HA patients (Keeney et al., 2005), so we screened for this mutation as the first step. Our study revealed that the prevalence of intron 22 inversion in mild HA patients in Sichuan was 44\% (8/18), which was still the most common genetic abnormality. Intron 1 inversion has been identified in Chinese patients with a prevalence of $1.9 \%$ in severe HA cases (Lu, et al., 2011), which was lower than in British people. We did not find intron 1 inversion in this study, possibly owing to the relatively small sample size.

Linkage analysis is the second most widely used method for carrier detection in HA families. Polymorphisms and STRs interspersed within the genome have been exploited in the diagnosis. The BclI polymorphic site in intron 18 , the HindIII site in intron 19, and 
dinucleotide CA-repeat STR markers in introns 13, 22 are the most common markers in use. We also selected two STR markers in introns 1 and 24. For linkage analysis, only the families with heterozygous mothers are suitable for carrier detection. Among the mothers tested, 26.3 and $15.8 \%$ were found to be heterozygous for BclI and HindIII polymorphic markers, respectively, and their families were diagnosed using those markers; in India, the percentages were 46.3 and 38\% (Chowdhury et al., 2003). Possibly these two polymorphism markers were less informative in Chinese people in Sichuan. In our study, HindIII displayed a lower heterozygosis rate than $B c l \mathrm{I}$ and was closely linked to it. This result suggests that HindIII does not need to be analyzed if $B c l$ I is adopted in Sichuan Chinese HA carrier detection. Of the STR markers, intron 13 was the most informative with a detection rate of $36.8 \%$, followed by intron $1(27.7 \%)$ and intron $22(10.5 \%)$. No family in our study could be diagnosed with intron 24. STR markers in intron 13 and intron 22 have been studied in Chinese people from Hong Kong; $58 \%$ of families could be analyzed in total, which was a little higher than in our study (47.3\%) (Yip et al., 1994). The STR marker in intron 1, which we first adopted to make carrier detections in Chinese HA families, has a higher rate than that in intron 22, and may be the second most informative marker for Chinese people from Sichuan. Owing to the small sample pool, further studies involving many more people should be instigated to determine whether these results are representative of all Chinese people. With the combined use of polymorphism and STR markers, HA carrier detection could be made in $88.9 \%(16 / 18)$ of HA families.

Among the seven HA families whose coding region of the FVIII gene was directly sequenced, five patients and certain of their female relatives had mutations detected, and in one family only the patient had a primary mutation but his mother and grandmother were normal at that site. All of the six mutations we detected were already included in the HAMSTeRS database. No mutation was detected in the other one HA patient or his family members. For this family, possibly a mutation in the non-coding region affected the expression of the FVIII gene.

In summary, our study suggests a strategy for HA carrier detection: intron 22 inversion should be analyzed first, the negative families for this mutation should then be subjected to linkage analysis, and those that cannot be analyzed by the above methods should be subjected to direct sequencing to find the mutation. Using a combination of the three methods, we successfully detected the carriers in the $18 \mathrm{HA}$ families in our study. Our cohort was a small group and a larger study with more patients would be required to establish a reliable prevalence of various mutations in Sichuan Chinese patients with HA. We anticipate that the procedure will be helpful for detecting carriers and affected fetuses.

\section{Conflicts of interest}

The authors declare no conflict of interest.

\section{ACKNOWLEDGMENTS}

Research supported by the Science and Technology Project of Sichuan (\#2010SZ0130).

\section{REFERENCES}

Bagnall RD, Waseem N, Green PM and Giannelli F (2002). Recurrent inversion breaking intron 1 of the factor VIII gene is a frequent cause of severe hemophilia A. Blood 99: 168-174.

Chowdhury MR, Tiwari M, Kabra M and Menon PS (2003). Prenatal diagnosis in hemophilia A using factor VIII gene 
polymorphism - Indian experience. Ann. Hematol. 82: 427-430.

Gitschier J, Wood WI, Goralka TM, Wion KL, et al. (1984). Characterization of the human factor VIII gene. Nature 312: 326-330.

He Z, Chen J, Xu S, Chen S, et al. (2013). A strategy for the molecular diagnosis in hemophilia A in Chinese population. Cell Biochem. Biophys. 65: 463-472.

Keeney S, Mitchell M, Goodeve A and UK Haemophilia Center Doctors' Organization Haemophilia Genetics Laboratory Network (2005). The molecular analysis of haemophilia A: a guideline from the UK haemophilia centre doctors' organization haemophilia genetics laboratory network. Haemophilia 11: 387-397.

Lakich D, Kazazian HH Jr, Antonarakis SE and Gitschier J (1993). Inversions disrupting the factor VIII gene are a common cause of severe haemophilia A. Nat. Genet. 5: 236-241.

Lalloz MR, McVey JH, Pattinson JK and Tuddenham EG (1991). Haemophilia A diagnosis by analysis of a hypervariable dinucleotide repeat within the factor VIII gene. Lancet 338: 207-211.

Liu Q, Nozari G and Sommer SS (1998). Single-tube polymerase chain reaction for rapid diagnosis of the inversion hotspot of mutation in hemophilia A. Blood 92: 1458-1459.

Lu YL, Wang XF, Ding QL, Dai J, et al. (2011). Prevalence of the factor 8 gene intron 1 inversion in Chinese haemophiliacs and its application to carrier detection and prenatal diagnosis in haemophilia A families. Haemophilia 17: 541-542.

Naylor J, Brinke A, Hassock S, Green PM, et al. (1993). Characteristic mRNA abnormality found in half the patients with severe haemophilia A is due to large DNA inversions. Hum. Mol. Genet. 2: 1773-1778.

Oldenburg J, Ananyeva NM and Saenko EL (2004). Molecular basis of haemophilia A. Haemophilia 10: 133-139.

Peake IR, Lillicrap DP, Boulyjenkov V, Briët E, et al. (1993). Report of a joint WHO/WFH meeting on the control of haemophilia: carrier detection and prenatal diagnosis. Blood Coagul. Fibrinolysis 4: 313-344.

Tasleem Raza S, Husain N and Kumar A (2009). Screening for hemophilia A carriers: utility of PCR-RFLP-based polymorphism analysis. Clin. Appl. Throm. Hemost. 15: 78-83.

Tizzano EF, Cornet M, Domènech M and Baiget M (2003). Exclusion of mosaicism in Spanish haemophilia A families with inversion of intron 22. Haemophilia 9: 584-587.

Yip B, Chan V and Chan TK (1994). Intragenic dinucleotide repeats in factor VIII gene for the diagnosis of haemophilia A. Br. J. Haematol. 88: 889-891. 